\title{
Absorption Enhancement of $a$-Si Thin Film Solar Cells through Surface Plasmon Polaritons and Cavity Resonance
}

\author{
Chuanhong Liu, Guangyao Su, Fangwang Gou, Zhaoyu Zhang* \\ School of Electronic and Computer Engineering, \\ Peking University Shenzhen Graduate School \\ Shenzhen, China \\ zhangzy@pkusz.edu.cn
}

\begin{abstract}
A back metallic binary-rectangle grating is proposed to realize absorption enhancement through introducing plasmonic modes and cavity resonance modes. The grating contains a secondary grating whose height is optimized. Adding the structure can enhance absorption by a factor of 2.6 at $\lambda=$ $915 \mathrm{~nm}$ and for the wavelengths in the range $550-790 \mathrm{~nm}, 875$ $900 \mathrm{~nm}$ the absorption is also enhanced.
\end{abstract}

Keywords: solar cell; plasmonic mode; cavity resonance;

\section{INTRODUCTION}

To meet the increasing demand of clean energy, many researches have been performed to develop efficient and low cost solar cells, among which, the silicon based solar cells is indispensable. Reducing solar cell active layer thickness not only decreases material consumption, but also increases the carrier collection rate, for which, thin film solar cells whose semiconductor layer is only several hundred nanometers causes much interest. However, the reduction of the thickness of the active layer may result in inefficient light absorption and low device efficiency. For this reason, increasing optical thickness of the device by light trapping is a fundamental issue in thin film solar cell design [1].

In the past few years, several techniques have been introduced to increase the light trapping in thin film solar cells. One of the most efficient methods is introducing nanostructures. For the metallic nanostructures placed at the top, bottom or buried inside the active layer, the enhancement of effective optical thickness is mainly due to the scattering, the excitation of surface plasmon polaritons and localized surface plasmons. For the dielectric particle and grating, the mechanisms are mainly scattering, diffraction, and coupling of waveguide modes. In order to find out the best structures ,a large number of research has been done, including the front gratings [2], the back gratings [3], the dual-gratings [4] and the photonic crystal. Besides, many researches focus on the arrangement of the particles, including Gaussian disorder surface [5] and gold angle arrangement surface [6].

However, in conventional designs, the enhancement for the long wavelengths is limited. In this work, we design and analyze rectangle-shaped nano metallic binary-grating on the silver (Ag) back contact numerically. We study the influence of the plasmonic modes and the cavity resonance induced by the grating and absorption enhancement is observed, especially for the long wavelengths.

\section{METHODS AND MODELS}

Calculations of the fields surrounding our models were done using finite element method (FEM) software from COMSOL Multiphysics4.2a. The light illumination on our structure is regarded as an incoming plane wave with wavelengths in the range $300-950 \mathrm{~nm}$; the sketch of the investigated structure is shown in Fig.1. It consists of a transparent conducting oxide layer made by indium tin oxide (ITO), whose thickness is $70 \mathrm{~nm}$ which is equivalent to $\lambda / 4 n$, for anti-reflection coating purpose, where $\lambda$ is the center wavelength and $n$ is the refractive index of ITO $(n=1.7)$. The absorption material is amorphous (a-Si) and is $100 \mathrm{~nm}$ thick. Rectangle binary-grating structure is introduced on the bottom Ag layer and the gap between the grating and the active layer is filled with ITO, to increase the carrier collection. The period is $640 \mathrm{~nm}$ which is twice of the optimization of a single grating, and the fill factor is 0.625 . The height of primary grating $(H \mathrm{c})$ is $100 \mathrm{~nm}$ and the width is $120 \mathrm{~nm}$.

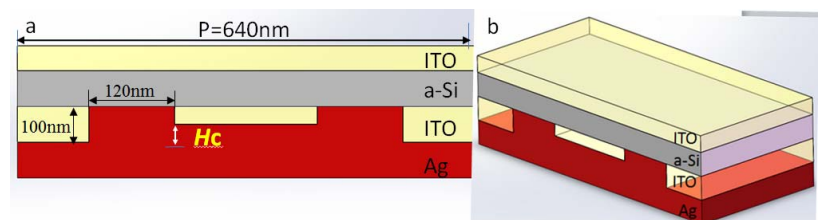

Fig.1. Schematic diagrams of the proposed solar cell structure.(a) A cross-sectional view outlining the device's structure to be used in simulations.(b) A 3D conceptual schematic.

\section{RESULTS AND DISCUSSIONS}

In order to quantify the absorption properties of the solar cell with the structure under non-polarized illumination, we calculate the average absorption with (1) [7]:

$$
A_{\text {average }}=\left(A_{T M}+A_{T E}\right) / 2
$$

where $A_{T M}$ and $A_{T E}$ are the total absorption for the TMpolarized and TE-polarized illumination respectively. Results 


\section{NUSOD 2013}

of the structure are presented in Fig.2 where the absorption enhancement is plotted as a function of the correlation length for several different values of $H c$ which means the height of the secondary-grating. Since we aim at the enhancement of the absorption, especially for the long wavelengths, only the results for the wavelength in range of $500-950 \mathrm{~nm}$ is shown. A planar solar cell is chosen as a reference.

It is obvious that there are several absorption enhancement peaks in the Fig.2 (a) and the enhancement is about 1.5 for the wavelengths range $650 \mathrm{~nm}$ to $720 \mathrm{~nm}$. The enhancement is close to 2.5 for the wavelengths near $900 \mathrm{~nm}$. Obviously, Fig.2 (b) shows a few areas in which large light absorption enhancement occurs. In order to find out the mechanisms for the enhancement, we choose the structure with $H \mathrm{c}$ equals 20 $\mathrm{nm}$ to analyze, which performances better compared to the other four structures.

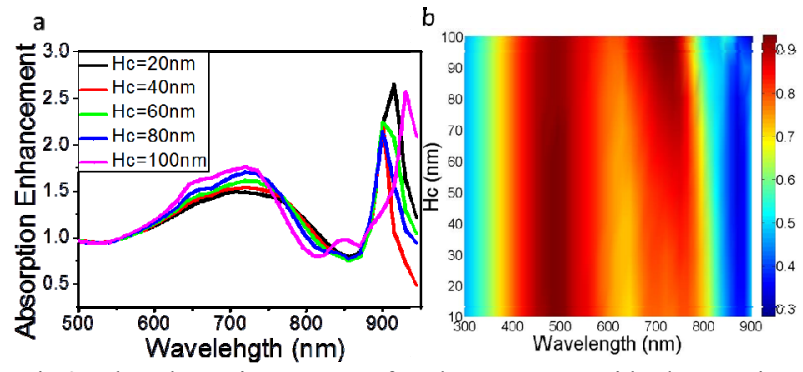

Fig.2 The absorption spectra for the structure with the varying secondary-grating height $H \mathrm{c}$.(a)The absorption enhancement spectra for $H \mathrm{c}=20 \mathrm{~nm}, 40 \mathrm{~nm}, 60 \mathrm{~nm}, 80 \mathrm{~nm}$ and $100 \mathrm{~nm}$.(b)The absorption efficiency as a function of both wavelength and height of the secondary-grating.

To find out the process of the enhancement, the EM field distribution across the cell is simulated, which shows strong inside the active layer. The first peak in Fig.2 (a), at $\lambda=680$ $\mathrm{nm}$, is identified as a product of both the SPP resonance and the Fabry-Pe'rot resonance of the film. This effect results in high coupling for the resonance mode, as the SPP mode is localized near its resonant frequency.

The enhancement corresponding to a wavelength of $900 \mathrm{~nm}$ becomes complicated after adding the nano gratings. Three absorption enhancement mechanisms can be identified. The first mechanism is related to cavity resonance and can be seen in Fig.3 (a) obviously. The interference with in the thin film leads to a resonant F-P cavity effect, depending on the thickness of the layer. Another resonance is introduced by adding the binary-gratings which is also shown in Fig. (a), whose mode strength is related to the parameter of the metallic back binary-grating. The second mechanism directly relates to SPPs that can be excited only by TM polarization, which can be inferred from the strong $\mathrm{Hz}$ field observation in Fig.3 (b). The third mechanism will be associated with the coupling into waveguide modes, and these modes can be found in nearly the middle of the active layer.

It is obvious that there is an enhancement peak shift in Fig.2 (a), which can be explained that the introduction of the binary- grating changes the effective thickness of the cavity [8] and brings about another cavity. This phenomenon is useful to shift the absorption peak to the wavelength where the absorption is poor, while the energy is rich under the AM1.5.

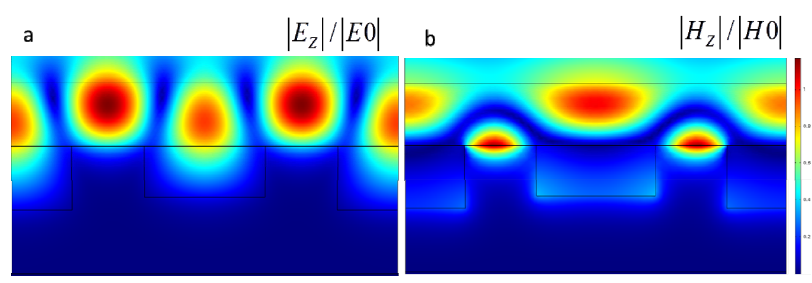

Fig.3. Normalized field intensity distribution at the wavelength 900 $\mathrm{nm}$.(a) For the incoming light is a TM-polarization beam having a wavelength at $900 \mathrm{~nm}$.(b) The incoming light is TE-polarization beam with the same wavelength as (a).

In conclusion, we successfully improve light absorption with back metallic binary gratings, especially the textured structure can significantly enhance light absorption for the wavelengths range $650 \mathrm{~nm}$ to $750 \mathrm{~nm}$ and $875 \mathrm{~nm}$ to $915 \mathrm{~nm}$ under the standard AM1.5 solar spectrum. The main mechanisms for the enhancement can be attributed to the excitation of plasmonic modes and cavity resonances. These properties of the metallic binary-grating are useful to improve the light absorption in thin film solar cells, especially for the long wavelengths.

\section{ACKNOWLEDGMENT}

This work was supported by Startup fund by Peking University Shenzhen Graduate School. The authors would like to acknowledge the valuable discussion with Xudong Liu, Deng Xiao, Ji Chen in the same group.

\section{REFERENCES}

[1] H. A. Atwater and A. Polman, "Plasmonics for improved photovoltaic devices," Nat Mater, vol. 9, pp. 205-13, Mar 2010.

[2] V. E. Ferry, L. A. Sweatlock, D. Pacifici, and H. A. Atwater, "Plasmonic Nanostructure Design for Efficient Light Coupling into Solar Cells," Nano Letters, vol. 8, pp. 4391-4397, Dec 2008.

[3] J. Gjessing, E. S. Marstein, and A. Sudbo, "2D back-side diffraction grating for improved light trapping in thin silicon solar cells," Opt Express, vol. 18, pp. 5481-95, Mar 152010.

[4] X. Meng, E. Drouard, G. Gomard, R. Peretti, A. Fave, and C. Seassal, "Combined front and back diffraction gratings for broad band light trapping in thin film solar cell," Opt Express, vol. 20 Suppl 5, pp. A56071, Sep 102012.

[5] P. Kowalczewski, M. Liscidini, and L. C. Andreani, "Engineering gaussian disorder at rough interfaces for light trapping in thin-film solar cells," Opt. Lett, vol. 37, pp. 4868-4870, 2012.

[6] J. Trevino, C. Forestiere, G. Di Martino, S. Yerci, F. Priolo, and L. Dal Negro, "Plasmonic-photonic arrays with aperiodic spiral order for ultrathin film solar cells," Opt Express, vol. 20, pp. A418-30, May 72012.

[7] R. B. Dunbar, T. Pfadler, and L. Schmidt-Mende, "Highly absorbing solar cells--a survey of plasmonic nanostructures," Opt Express, vol. 20 Suppl 2, pp. A177-89, Mar 122012.

[8] W. Wang, S. Wu, K. Reinhardt, Y. Lu, and S. Chen, "Broadband Light Absorption Enhancement in Thin-Film Silicon Solar Cells," Nano Letters, vol. 10, pp. 2012-2018, 2010/06/09 2010. 